\title{
Structural Damage Detection Using Artificial Neural Networks and Wavelet Transform
}

\author{
Arthur Shi, Xiao-Hua Yu
}

\begin{abstract}
With the ever-increasing demand for the safety and functionality of civil infrastructures, structure health monitoring (SHM) has now become more and more important.

Recent developments in computational intelligence and digital signal processing offer great potentials to develop a more efficient, reliable, and robust structure damage identification system. In this paper, the application of artificial neural networks and wavelet analysis is investigated to develop an intelligent and adaptive structural damage detection system. The proposed approach is tested on an IASC (International Association for Structural Control)-ASCE (American Society of Civil Engineers) SHM benchmark problem. Satisfactory computer simulation results are obtained.
\end{abstract}

\section{INTRODUCTION}

Civil infrastructure plays an important role in a nation's economic prosperity and has a great impact on our daily lives. With the ever-increasing demand for the safety and functionality of civil infrastructures, structure health monitoring (SHM) has become more and more important.

In general, structural damages can be detected by routine check-ups via human visual inspections. This traditional, laborintensive method requires large amount of manpower, and it is only effective in detecting severe damages that are visible and near the structure surface. In a modern non-destructive evaluation (NDE) SHM system, the raw measurement data from the wireless sensors that are mounted on buildings or bridges are automatically transmitted to a central information processing unit in real-time. The information processing unit (which is the most important component of the SHM system) determines the status or the health condition of infrastructures based on the analysis of the data stream from sensors ([1]). In fact, the major component of the information processing unit is a signal assessment algorithm.

There are two distinctly different approaches for real time automatic structure damage detection; i.e., the model based approach and the non-model (or model-free) approach. In the former, a precise mathematical model of the civil infrastructure must be developed in advance, usually with the assumption that the system under study is linear and time-invariant. This approach is based on the fact that any physical structural damage often results in changes in system parameters and shift of pole locations in complex plane. Damage diagnosis can then be performed by studying the changes of the structure's dynamic characteristics such as natural frequency, stiffness, and damping ratio, etc. ([2]) A similar and related method is to identify the pole locations of the transfer function of the structure in current status and then compare them with the ones of the same structure in intact mode ([3]).

The model-free approach does not require a priori knowledge on the structure's parameters; instead, it directly relates the sensor data from an instrumented structure with the causes and locations of damage patterns ([4]). It is known that structural damage results in changes on structure vibration dynamics which can be recorded by various types of sensors, including strain gages, accelerometers, and displacement transducers. Many damage detection studies focus on the analysis of the time history data collected from sensor measurement ([5], [6], and [9]). This approach does not rely on an accurate mathematical model so it is more flexible and suitable for real-time damage detection. However, how to extract the "best" (or the most appropriate) feature from the measurement time history data still remains as an open question. In addition, the short-term and non-stationary nature of the system transient response requires advanced signal processing techniques to characterize and classify different damage patterns.

In modern signal processing, wavelet transform is a mathematical tool for multi-resolution analysis. Multiresolution analysis can analyze the different frequency components of a signal with different levels of details, and thus is an effective way to extract information from non-stationary signals. Recently, wavelet transform has been employed to identify the singularity (abnormality) of structural dynamic responses ([5], [6]) and vibration mode shapes ([7]). Wavelet analysis is well suited for the application of SHM to identify sudden changes or spikes in vibration signals. With multiresolution, damages can be more accurately located by analyzing the signal waveforms in a finer scale; and the severities of damages can be further identified by comparing the values of their wavelet transform coefficients. Also, measurement data is often contaminated with noise, which makes damage detection very difficult. An additional advantage of wavelet transform is that the thresholding technique (also called wavelet shrinkage) can be applied on wavelet coefficients for noise reduction. However, the "direct" wavelet decomposition for damage detections may still fail to respond when the measurement signal is corrupted with strong interference and/or noise, i.e., with a low signal-to-noise ratio 
(SNR), especially when the structural change or damage is gradual.

Artificial neural networks (ANN) have been successfully applied to solve pattern recognition and classification problems in recent years. ANN has many important properties, such as adaptive learning, distributed association, and nonlinear mapping. In ANN, learning is achieved by adjusting the connections (weights) in the network to minimize a specific performance index (e.g., the mean square error at its output).

Recently, artificial neural networks have also been employed to detect damage for structural health monitoring. In [4], a multi-layer feedforward neural network is trained to relate the frequency response function (FRF) characteristics and the damage location/severity.

In this research, we propose a hybrid damage detection scheme which combines wavelet analysis and artificial neural networks together to improve the reliability of an adaptive structure health monitoring system. Wavelet transform is employed to extract the underlying features of the transient response from measurement data; then these feature vectors are employed as the input of the artificial neural network in which damage patterns can be further identified and classified. The proposed approach is applied and tested on an IASC (International Association for Structural Control) - ASCE (American Society of Civil Engineers) SHM benchmark problem via computer simulation.

The rest of the paper is organized as follows. First, a brief discussion on wavelet analysis is given in section 2; then the wavelet-neural network approach for structural damage detection is presented in section 3. In section 4, this approach is applied to the IASC-ASCE benchmark structure and computer simulation results are presented. Section 5 concludes the paper and also gives directions for future works.

\section{THE WAVELET TRANSFORM}

Wavelet analysis is a mathematical tool that decomposes data into different frequency components, and then studies each component with a different (time) resolution ([8]). The wavelet transform can be reviewed as the inner product, or the crosscorrelation of a signal with a set of wavelets of various "widths" and scales. These wavelets are generated from a single function (known as the "mother wavelet") with different translation (or shift) factors/coefficients and dilation (or scale) factors/coefficients. Wavelet analysis has many advantages over traditional Fourier methods due to its multi-resolution property, especially when analyzing signals that contain discontinuities and/or sharp spikes.

A mother wavelet function $\psi(t)$ is a basis function with finite energy function that should satisfy the two following criteria:

$$
\int_{-\infty}^{\infty} \psi(t) d t=0
$$

and

$$
\int_{-\infty}^{\infty}|\psi(t)|^{2} d t<\infty
$$

The continuous wavelet transform (CWT) of a function $f(t)$ is defined as:

$$
\Psi_{\psi}[f(s, \tau)]=\int_{-\infty}^{\infty} f(t) \psi_{s, \tau}^{*}(t) d t
$$

where $\psi_{s, \tau}(t)$ represents the family of wavelets and the "*" represents the operation of complex conjugate:

$$
\psi_{s, \tau}(t)=\frac{1}{\sqrt{|S|}} \psi\left(\frac{t-\tau}{s}\right)
$$

where $\tau$ is the dilation coefficient and $s$ is the translation coefficient.

For computational purpose, discrete wavelet transform (DWT) is often used. The family of discrete wavelets $\psi_{m, n}(t)$ can be written as:

$$
\psi_{m, n}(t)=2^{-m / 2} \psi\left(2^{-m} t-n\right)
$$

where $m$ and $n$ are integers for indices.

DWT can be implemented using a series of high- and lowpass filters. When the signal passes through a high-pass filter $h$, it yields detail coefficients. When it passes through a lowpass filter $g$, it yields approximation coefficients. The level of decomposition can be repeated, as shown in Fig. 1.

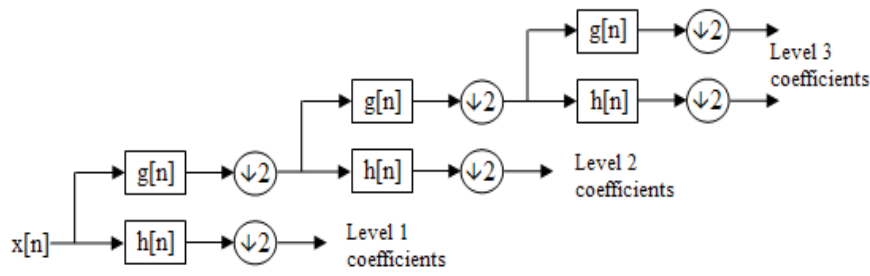

Fig. 1. The discrete wavelet transform (DWT) ([11])

The most commonly used set of discrete wavelet transforms is the Daubechies wavelets ([12]). Daubechies wavelets have been widely used in solving a broad range of problems and are especially suitable for detecting signal discontinuities. In this research, Daubechies 4-tap wavelets (D4) is employed (Fig. 2).

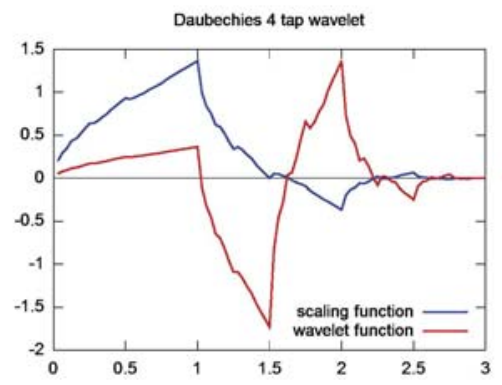

Fig. 2. The Daubechies 4-tap (D4) wavelets ([12]) 


\section{THE WAVELET-NEURAL NETWORK APPROACH}

The overall proposed structure health monitoring system is shown in Fig. 3. First, raw measurement data from sensors are obtained and pre-processed. This process includes data interpolation and/or re-sampling if needed, as well as basic filtering techniques (e.g., high-pass or low-pass filters) for noise reduction. After pre-processing, wavelet transform is applied to extract features from vibration signals. By appropriate thresholding, the noise and interferences in the signal can be further reduced. The feature vectors (wavelet coefficients) are then become the inputs of an artificial neural network which determines whether the structure is damaged. Note that with the application of ANN, the proposed intelligent SHM system yields an important property of adaptive learning; that is, it can learn, or update itself, from new sensor measurements continuously if desired.

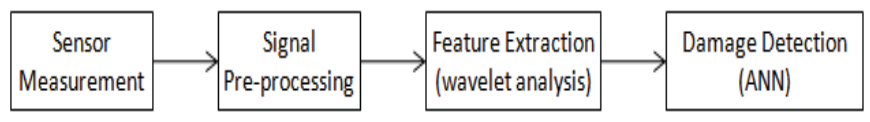

Fig. 3. The wavelet-neural network approach

A multi-layer feedforward neural network is employed in this research. All the weights are updated using the Levenberg-Marquardt algorithm to minimize the following objective function:

$$
J=\frac{1}{2 N} \sum_{i=1}^{N}[e(i)]^{2}=\frac{1}{2 N} \sum_{i=1}^{N}[d(i)-y(i)]^{2}
$$

where $d$ is the desired output and $y$ is the output of neural network; $e$ is the output error; $i$ is the index of training pair; and $N$ is the batch size ( $N=1$ for online or incremental training).

$$
\begin{aligned}
& W(k+1)=W(k)+\Delta W \\
& \Delta W=\left(J_{a}{ }^{T} J_{a}+\mu I\right)^{-1} J_{a}{ }^{T} e
\end{aligned}
$$

where $J_{a}$ is the first order derivative of the error function with respect to the neural network weight matrix (also called the Jacobian matrix) $\frac{\partial e}{\partial W} ; \mu$ is a learning parameter; and $k$ is the index of iterations.

\section{COMPUTER Simulation RESUlts}

In this research, the proposed approach is applied and tested on an IASC (International Association for Structural Control) ASCE (American Society of Civil Engineers) SHM benchmark problem. This benchmark study was conducted by the IASCASCE SHM Task Group based on a laboratory scale prototype structure, which is a four-story steel-frame quarter-scale model in the Earthquake Engineering Research Laboratory at the University of British Columbia (Fig. 4). Finite element models based on this structure were derived to generate the simulated response data, which can then be used as a basis of comparison to test different SHM systems. In addition to the undamaged structure, the benchmark problem also includes six different damage patterns ([10]). This benchmark study provides a testbed for comparing various damage detection techniques and has been employed by many researchers in recent years ([6], [9]).
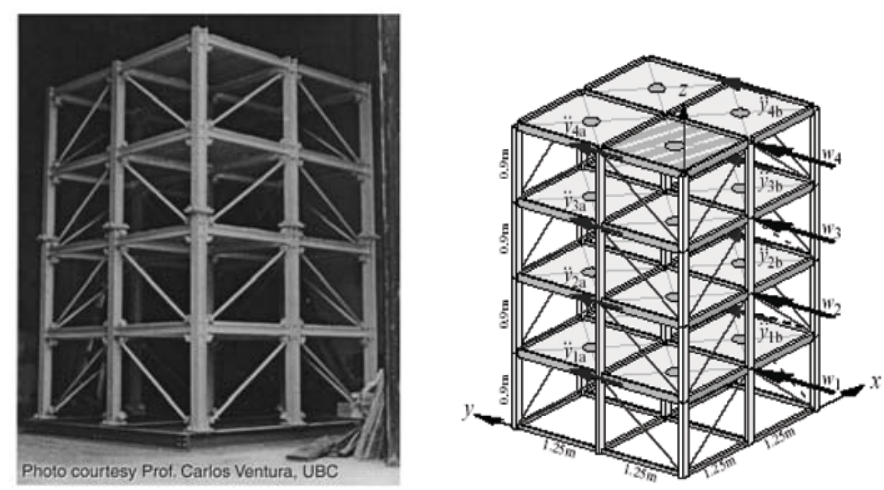

Fig. 4. The IASC-ASCE SHM benchmark model ([10])

\section{A. Signal Decomposition and Wavelet Coefficients}

The first row in Fig. 5 shows the signal measured by the acceleration sensor when beam 4 of the structure is damaged (the total signal time duration is 4 seconds and the damage occurs at about $t=2$ second). The raw sensor signal is contaminated with Gaussian white noise (due to wind); thus a sixth order Butterworth low-pass filter with a cut-off $(3 \mathrm{~dB})$ frequency of $100 \mathrm{~Hz}$ is employed for pre-filtering. Obviously, it is difficult to detect damage by observing the waveform (in the first row of Fig. 5) directly without any signal processing technique.

The next 6 rows in Fig. 5 show the Daubechies (D4) wavelet transform decomposition at different levels. The last (the $7^{\text {th }}$ ) row shows the level 1 detail coefficients; while the $6^{\text {th }}$, $5^{\text {th }}$, and $4^{\text {th }}$ row shows the detail coefficients of level 2,3 , and 4 , respectively. Note the detail and approximate coefficients of level 5 are shown in the $2^{\text {nd }}$ and the $3^{\text {rd }}$ row; and we can conclude from Fig. 5 that the approximate coefficients of level 5 decomposition are suitable for damage detection.

\section{B. The Neural Network Configuration}

To determine the appropriate size of the neural network, various tests are conducted. In this research, only one output neuron is needed to indicate whether there is a structural damage (" 1 " for the case of damage and " 0 " for the undamaged case, with a threshold of 0.5). However, the number of inputs, the number of hidden layers and the number of neurons in each hidden layer may vary.

The inputs of the neural network are the level 5 wavelet transform coefficients which must be in the power of 2. Four different networks are tested; each one of them has 8, 16, 24, and 32 inputs, respectively. Although the network with larger number of inputs seem to yield higher accuracy during training phase, the improvement is not significant and the 
MSE (mean-square-error) in testing phase may even increase an indication of over-fitting. It is observed that the network with 16 inputs yields a low MSE during training and the lowest MSE in testing phase; therefore we choose the number of inputs to be 16 .

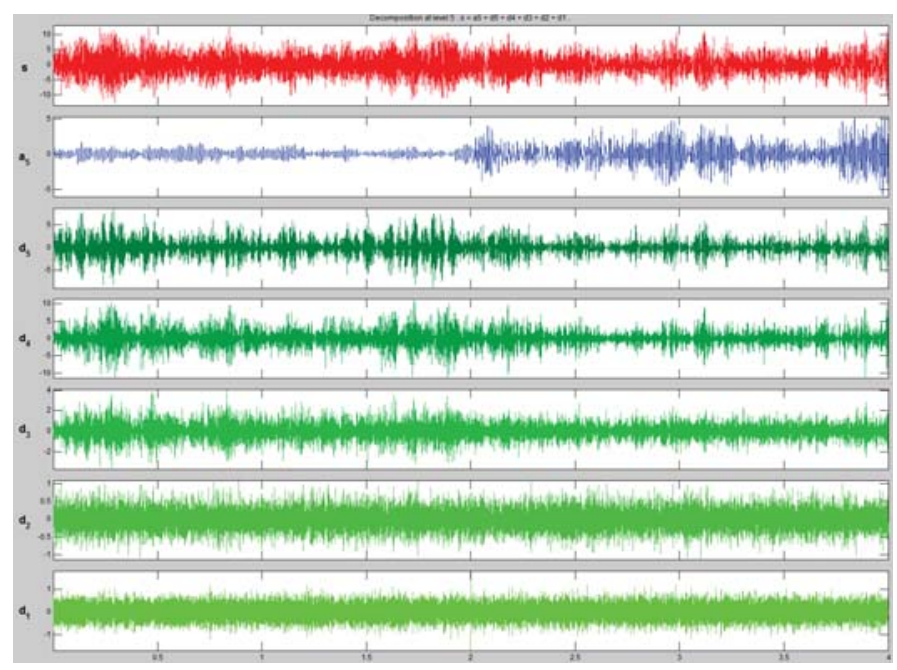

Fig. 5. Wavelet coefficients at different levels

Next, the number of hidden neurons needs to be determined. A network with one hidden layer is considered here and the number of hidden neurons is varied (considering the case of 10, 20,40, and 80 hidden neurons). Computer simulation results show that the network with 20 hidden neurons results a low training MSE and the best testing MSE.

Finally, the optimal number of hidden layers needs to be determined. We consider the case of 1, 2, 4, and 8 hidden layers (with 20 hidden neurons in each layer). Simulation results show that when there are 4 hidden layers in the network, the network yields the lowest MSE in both training and testing phases.

From the above discussion, we conclude the most appropriate size of neural network for this research is a feedforward network with 4 hidden layers and 20 hidden neurons in each layer. The activation function of the output neuron is linear while the activation function of hidden neurons is the hyperbolic tangent function:

$$
f(x)=\frac{1-e^{-x}}{1+e^{-x}}
$$

When training the neural network, a "sliding window" technique is employed. That is, after the first set of data points is used to find wavelet coefficients, the "window" (with the same size) is shifted by $K$ points to obtain the next set of data points for wavelet decomposition (overlapping with the previous data sets). This ensures that there is enough data for both training and testing of the neural network.

\section{C. $\quad$ Test Results}

The above "optimized" neural network now can be applied for damage detection. To evaluate the performance of the wavelet-neural network approach, two different damage cases are considered, i.e.,

Case 1: All braces on 1st story broken

Case 2: All braces on 1st and 3rd story broken

The simulation results are listed in Table 1. In training phase of damage case 1, among 621 different damaged/undamaged data sets (about 50\% each, i.e., 311 undamaged sets and 310 damaged sets), only 3 of them are misclassified; in other words, the correct classification rate is $99.52 \%$. Once the neural network is fully trained, its performance is tested on a different dataset (also about $50 \%$ each of damaged/undamaged data sets). In testing phase, 28 out of 621 sets are misclassified; that is, the correct classification rate is about $95.49 \%$. Similar results can be obtained with damage case 2, with the percentage of correct classification of $99.03 \%$ for training and $96.78 \%$ for testing. From these results, we conclude that the proposed waveletneural network approach can successfully detect structural damage.

Table 1. Test Results

\begin{tabular}{|c|c|c|c|c|}
\hline \multirow{2}{*}{ Case } & \multicolumn{2}{|c|}{ Training } & \multicolumn{2}{c|}{ Testing } \\
\cline { 2 - 5 } & $\begin{array}{c}\text { Incorrect } \\
\text { classification }\end{array}$ & $\begin{array}{c}\% \\
\text { Correct }\end{array}$ & $\begin{array}{c}\text { Incorrect } \\
\text { classification }\end{array}$ & $\begin{array}{c}\% \\
\text { Correct }\end{array}$ \\
\hline 1 & 3 & 99.52 & 28 & 95.49 \\
\hline 2 & 6 & 99.03 & 20 & 96.78 \\
\hline
\end{tabular}

\section{Discussion}

The wavelet transform in this approach serves for two purposes. It not only provides inputs to the neural network, but also reduces noise (via thresholding). The neural network performs the task of pattern recognition (damage detection); in addition, its fault tolerance and extrapolation ability also help to further attenuate the effect of noise in sensor measurement data. The simulation results show the combined waveletneural network approach is promising and has great potentials for structural damage detection.

\section{CONCLUSIONS AND FUTURE WORKS}

In this paper, a hybrid wavelet-neural network approach for structural damage detection is discussed and applied to an IASC-ASEE benchmark structure. Computer simulation results show this approach can successfully detect damage with satisfactory accuracy $(95.49 \%$ and $96.78 \%$ for the two testing damage patterns). Further evaluation and testing (on more damage patterns) will be conducted.

\section{REFERENCES}

[1] Lynch, J. \& Loh, K. 2006. A Summary Review of Wireless Sensors and Sensor Networks for Structural Health Monitoring. Shock and Vibration Digest, 38 (2), pp. 91-128.

[2] Salawu, O. 1997. Detection of Structural Damage Through Changes in Frequency: a Review. Engineering Structures, 19 (9), pp. 718-723. 
[3] Lynch, J. 2005. Damage Characterization of the IASC-ASCE Structural Health Monitoring Benchmark Structure by Transfer Function Pole Migration. Proceedings of the 2005 Structures Congress and the 2005 Forensic Engineering Symposium.

[4] Fang, X. \& Luo, H. et al. 2005. Structural damage detection using neural network with learning rate improvement. Computers and Structures, 83, pp. $2150-2161$.

[5] Hou, Z. \& Noori, M. et al. 2000. Wavelet-based approach for structural damage detection. Journal of Engineering Mechanics, 126 (7), pp. 677683.

[6] Hou, Z. \& Hera, A. 2002. Progress of Phase II Study of the ASCE Health Monitoring Benchmark Data Using Wavelet Approach. Proceedings of the 15th ASCE Engineering Mechanics Conference.
[7] Hou, Z. \& Hera, A. et al. 2006. Wavelet-Based Structural Health Monitoring of Earthquake Excited Structures. Computer-Aided Civil and Infrastructure Engineering, 21(4), pp. 268-279.

[8] Graps, A. 1995. An Introduction to Wavelets. Computational Science \& Engineering, 2 (2), pp. $50-61$.

[9] Lin, S. \& Yang J. et al. 2005. Damage identification of a benchmark building for structural health monitoring. Smart Materials and Structures, pp. S162-S169.

[10] Johnson, E. \& Lam, H. et al. 2004. Phase I IASC-ASCE Structural Health Monitoring Benchmark Problem Using Simulated Data. Journal of Engineering Mechanics, 130 (1), pp. 3-15.

[11] http://en.wikipedia.org/wiki/Discrete wavelet transform

[12] http://en.wikipedia.org/wiki/Daubechies_wavelet 